\title{
ON THE BOUNDARY VALUES OF RIEMANN'S MAPPING FUNCTION
}

\author{
BY
}

R. J. V. JACKSON

\begin{abstract}
Classically, the calculus of variations is required to prove the existence of a biholomorphism from the unit disk to a given simply-connected, smooth domain in the complex plane. Here, the problem is reduced to the solution of an ordinary differential equation along the boundary of the domain. The sole coefficient in this equation is identified with the bounded term in the asymptotic expansion of the Bergman kernel function. It is shown that this coefficient can not depend upon any differential expression involving only the curvature function of the boundary.
\end{abstract}

0. Introduction. Suppose $\Omega$ is a bounded, simply-connected, smooth domain in the complex plane, equipped with an arc length parameterization $\phi(s)$ for its boundary $\Gamma=b \Omega$ which encircles the interior in a counterclockwise direction. It has been known for a long time that there exists a bijective holomorphic function $F(z)$ from $\Omega$ to the unit disk $B$; in fact, there is a family of such functions which depends on three real parameters (they are the Riemann mapping functions; here, they will be termed biholomorphisms). The existence of these mappings can be proven in several ways; but all the methods are purely theoretical in that each obtains the biholomorphism as the solution to some problem in the calculus of variations. Except for very special domains, one is unable to actually write down the required biholomorphism in general. This article seeks to make the construction of $F(z)$ more explicit; the idea centers around the following fact: when the boundary $\Gamma$ is smooth, the biholomorphism induces a smooth function $\theta=f(s)$ of the boundaries via the relation

$$
F(\phi(s))=e^{i f(s)} .
$$

We shall reverse the procedure by constructing $F(z)$ from its boundary function $f(s)$, the main issue being to distinguish the latter from all other possible mappings from $\Gamma$ to $S^{1}$. This will be done by characterizing the boundary function $f(s)$ in terms of solutions to an ordinary differential equation

$$
P_{\Gamma}(y) \equiv y^{\prime \prime}+\frac{1}{2} \gamma(s) \cdot y=0 .
$$

The zero-order coefficient of this equation can be identified with a certain diagonal term in the asymptotic expansion of the Bergman kernel $K_{\Omega}(z, \bar{\omega})$ for the domain $\Omega$; setting $z=\phi(s)$ and $\omega=\phi(r)$ so that $z^{\prime}=\phi^{\prime}(s)$ and $\omega^{\prime}=\phi^{\prime}(r)$, we have

Received by the editors June 27, 1978 and, in revised form, July 31, 1979.

AMS (MOS) subject classifications (1970). Primary 30A30, 30A88, 34B05; Secondary 30A31, $30 \mathrm{~A} 38$.

Key words and phrases. Riemann's mapping function, Plateau's problem, ordinary differential equations, potential theory, Hilbert's transform, asymptotics of Bergman's kernel function. 


$$
\frac{1}{6} \gamma(s)=\lim _{r \rightarrow s}\left[-\pi K_{\Omega}(z, \bar{\omega}) z^{\prime} \bar{\omega}^{\prime}-(s-r)^{-2}\right] .
$$

MAIN TheORem. $f: \Gamma \rightarrow S^{1}$ is the boundary value of some biholomorphism from $\Omega$ to $B$ if and only if the following statements are valid in the arclength parameter on $\Gamma$ :

(i) $f$ is orientation preserving, that is, $f^{\prime}(s)>0$.

(ii) It is given explicitly by $f(s)=2 \arctan (\tau(s) / \beta(s))$ where $\tau(s)$ and $\beta(s)$ are linearly independent solutions of the boundary value problem

$$
y^{\prime \prime}+\frac{1}{2} \gamma(s) y=0, \quad y(0)+y(L)=0, \quad y^{\prime}(0)+y^{\prime}(L)=0,
$$

on the interval from 0 to $L \equiv$ length of $\Gamma$.

The first condition is a simple consequence of our intention to map the interior of $\Omega$ onto the interior of the unit disk. The appearance of the function $2 \arctan (x)$ in the second condition is also relatively innocuous. It is the boundary function for $z=(i-\omega) \cdot(i+\omega)^{-1}$ which maps the upper half-plane $\mathbf{U}$ onto the unit disk. Thus the ratio $\tau(s) / \beta(s)$ will actually provide a mapping from $\Omega$ to $U$. The advantage of this description is twofold. First, one can calculate $f(s)$ by known iterative techniques; second, the boundary regularity of the biholomorphism is an immediate result of standard theorems about ordinary differential equations.

Although arclength was employed in the theorem, it is not necessary to restrict oneself to this parameter when calculating the boundary function. This has practical value because it is extremely difficult to obtain this parameter in many cases. For instance, in dealing with the ellipse $x^{2} / a^{2}+y^{2} / b^{2}<1$, the conversion from the obvious parameterization $x=a \cos \theta, y=b \sin \theta$ to the arclength involves the solution of an "elliptic" integral. This problem can be circumvented because the special coefficient $\gamma_{\Gamma}$ behaves in a very particular manner under coordinate transformations; in any parameter $t$, it is represented by some function $\tilde{\gamma}(t)$ which is related to the original expression $\gamma_{\Gamma}(s)$ via the identity

$$
\tilde{\gamma}(t)=\gamma_{\Gamma}(h(t)) \cdot\left[h^{\prime}(t)\right]^{2}+S_{h}(t)
$$

where $s=h(t)$ is the coordinate change on $\Gamma$, and $S_{h}$ is its Schwarzian derivative (which depends upon $h^{\prime}$ only, and thereby avoids the aforementioned problem on the ellipse). Moreover the operator $P_{\Gamma}$ is given in the new coordinates by the formula $\ddot{y}+\frac{1}{2} \tilde{\gamma}(t) \cdot y=0$. This aspect will be discussed in $\S 1$, but, in passing, we mention another interpretation. The biholomorphisms of $\Omega$ to itself form a real Lie group of three dimensions, and it is possible to view this group as a principal bundle over the boundary curve $\Gamma$. This embues $\Gamma$ with a kind of geometric structure, which bears a strong resemblence to the more well-known Riemannian geometry. For example, the coefficient $\gamma_{\Gamma}$ is akin to the set of Christoffel symbols, and the differential operator $P_{\Gamma}$ plays the role of a covariant derivative. The analog persists into the realm of several complex variables, for these objects are implicit in the pseudoconformal geometry of nondegenerate hypersurfaces; see reference [3]. The viewpoint of principal bundles would lead naturally to the existence and uniqueness of the special coefficient $\gamma_{\Gamma}$, however, it does not provide any other information about $\gamma_{\Gamma}$. In $\S 1$, this is remedied by an application of potential theory, 
as developed by Schiffer in [4] for example. The work will allow us to verify equation (0.2), and establish the necessity of the two conditions in the main theorem.

In proving that the conditions of the main theorem are also sufficient, the major task is to show that the function $f: \Gamma \rightarrow S^{1}$ extends to a holomorphic function on the interior $\Omega$; the bijectivity is then a routine matter to check. Equivalently, it suffices to show that the inverse map $g: S^{1} \rightarrow \Gamma$ extends to a holomorphic function on $B$. To this end, we shall view the operator $P_{\Gamma}$ as acting on the set of smooth functions $y(s)$ over $\Gamma$. As such, it possesses an inverse operator given by

$$
\left.Q y\right|_{s}=\int_{\Gamma} E_{\Gamma}(s, r) y(r) d r
$$

where $E_{\Gamma}(s, r)$ may be termed the Green's function for $P_{\Gamma}$. Due to the properties of $\gamma_{\Gamma}$, it is a remarkable fact that $E_{\Gamma}(s, r)$ is related to the boundary values of Bergman's kernel via the identity

$$
\frac{1}{E_{\Gamma}(s, r)^{2}}=-4 \pi K_{\Omega}(z, \bar{\omega}) z^{\prime} \bar{\omega}^{\prime}
$$

where as previously, $z=\phi(s)$ and $\omega=\phi(r)$. This identity should be useful in other contexts because it means that $K_{\Omega}(z, \bar{\omega})$ is completely determined by its diagonal value $\gamma_{\Gamma}(s)$ (for example, one might employ the fact that Green's function can be expanded in a series whose terms involve the eigenfunctions for the operator $P_{\Gamma}$ ). In the present context, this identity will be utilized by building upon the work of Douglas [6] concerning Plateau's problem. He introduced the integral functional

$$
A(h)=\frac{1}{16 \pi} \int_{0}^{2 \pi} \int_{0}^{2 \pi} \frac{|h(\theta)-h(\phi)|}{\sin ^{2}((\theta-\phi) / 2)} d \theta d \phi
$$

which operates on a suitably large class of reparameterizations $h: S^{1} \rightarrow \Gamma$. It follows from his development that any minimum for this functional must extend to a holomorphic function on $B$. In $\S 3$, we shall add to Douglas' work in two ways: first by showing that the function $h(\theta)=f^{-1}(\theta)$ provides an explicit minimum for this functional; the second, by presenting a direct proof that this minimum extends to a holomorphic function.

Finally let us return to the issue of explicitly constructing the biholomorphism $F$ : $\Omega \rightarrow B$. The problem is now reduced to finding the special coefficient $\gamma_{\Gamma}$. The appendix demonstrates that $\gamma$ cannot be a local invariant of the boundary curve $\Gamma=b \Omega$, that is, it cannot be found simply by knowing the curvature function $K_{\Gamma}(s)$ or its derivatives. This is one way to express the nonexplicit nature of Riemann's mapping function. The author hopes to devote a future article to the determination of $\gamma_{\Gamma}$; for the present, here is an open question: given a bounded $\Omega$ in $\mathbf{C}^{\mathbf{l}}$ with a smooth boundary, it is possible to devise a convergent iterative procedure which generates the special coefficient $\gamma_{\Gamma}$ from the curvature function $K_{\Gamma}$ ?

1. Necessity. The Schwarzian derivative will be employed extensively in the subsequent development. For the reader's convenience, we here summarize some of its most useful properties. If $f(x)$ is a smooth function with nonvanishing first 
derivative, its Schwarzian derivative is defined to be

$$
\left.S f\right|_{x} \equiv \frac{f^{\prime \prime \prime}(x)}{f^{\prime}(x)}-\frac{3}{2}\left[\frac{f^{\prime \prime}(x)}{f^{\prime}(x)}\right]^{2}
$$

This special combination can be simplified by an algebraic substitution:

$$
y=1 / \sqrt{f^{\prime}(x)} \text { satisfies the equation } y^{\prime \prime}+\frac{1}{2} S f \cdot y=0 .
$$

In other words, the Schwarzian is nonlinear, but it can be handled by linear techniques.

FACT 1.3. The Schwarzian $S f(x)=\sigma(x)$ if and only if $f(x)=\tau(x) / \beta(x)$ where $\beta(x)$ and $\tau(x)$ are uniquely prescribed solutions of $y^{\prime \prime}+\frac{1}{2} \sigma \cdot y=0$.

It is a matter of elementary calculation to show that the ratio $\tau / \beta$ has the Schwarzian $S(\tau / \beta)=\sigma$. The converse is more revealing. By equation (1.2), we see that $f^{\prime}(x)=\beta(x)^{-2}$ where $\beta(x)$ is some (nonvanishing) solution of the equation $y^{\prime \prime}+\frac{1}{2} \sigma \cdot y=0$. By the standard theory of ordinary differential equations, we may construct a second solution $\tau(x)$ which is linearly independent of $\beta(x)$. In fact, their Wronskian matrix

$$
\left[\begin{array}{ll}
\beta(x) & \tau(x) \\
\beta^{\prime}(x) & \tau^{\prime}(x)
\end{array}\right]
$$

can be normalized to have determinant 1 for all $x$. As proof, differentiate the determinant formula, and use the condition $y^{\prime \prime}+\frac{1}{2} \sigma \cdot y=0$ to show that the result is zero. Notice that for a given $x_{0}$, this places one linear relation on the numbers $\tau\left(x_{0}\right)$ and $\tau^{\prime}\left(x_{0}\right)$. Moreover, the ratio $p(x)=\tau(x) / \beta(x)$ now has derivative $p^{\prime}(x)=$ $[\beta(x)]^{-2}=f^{\prime}(x)$. By choosing the value of $\tau\left(x_{0}\right)$ so that $f\left(x_{0}\right)=p\left(x_{0}\right)$, we completely determine the solution $\tau(x)$, and simultaneously insure that $f(x)=$ $\tau(x) / \beta(x)$. Q.E.D.

Whenever the composition $h=g \circ f$ is defined, there is a "pseudogroup" rule which relates the Schwarzians of all three functions,

$$
S h=S g \cdot\left(f^{\prime}\right)^{2}+S f,
$$

the term $S g$ being evaluated at $y=f(x)$, all the others at $x$. These facts will be applied both when the function $f(x)$ is real- or complex-valued, and when it depends smoothly on a real variable, or holomorphically upon a complex variable.

To motivate the role of the Schwarzian derivative, we consider the real line $\mathbf{R}$ as the boundary of the upper half-plane $U=\{x+i y: y>0\}$. It is well known that the group of biholomorphisms from $U$ to $U$ is in $1: 1$ correspondence with the matrix group SL(2). The identification is accomplished by

$$
F_{M}(z)=\frac{a z+b}{c z+b} \quad \text { where the matrix } M=\left[\begin{array}{ll}
a & b \\
c & d
\end{array}\right]
$$

has determinant equal to one.

Given an arbitrary diffeomorphism $f(x)$ of $\mathbf{R}$, how well can it be approximated by the boundary values of these biholomorphisms? Because SL(2) depends on three 
real parameters, it is possible to obtain a third order approximation locally. With more precision, let $f: \mathbf{R} \rightarrow \mathbf{R}$ satisfy $f^{\prime}\left(x_{0}\right)>0$; then there is a unique matrix $M$ in SL(2) such that

$$
F_{M}^{-1} \circ f(x)=\left(x-x_{0}\right)+\vartheta\left|x-x_{0}\right|^{3} .
$$

The coefficients of the matrix $M$ depend upon the second derivatives of $f(x)$ at the point $x_{0}$ in accordance with the formula

$$
M\left(x_{0}\right)=\left[\begin{array}{cc}
1 & f\left(x_{0}\right) \\
0 & 1
\end{array}\right] \cdot\left[\begin{array}{cc}
\sqrt{f^{\prime}\left(x_{0}\right)} & 0 \\
0 & \frac{1}{\sqrt{f^{\prime}\left(x_{0}\right)}}
\end{array}\right] \cdot\left[\begin{array}{cc}
1 & 0 \\
-\frac{1}{2} \frac{f^{\prime \prime}\left(x_{0}\right)}{f^{\prime}\left(x_{0}\right)} & 1
\end{array}\right] .
$$

The leading term of the error turns out to depend only upon the Schwarzian derivative of $f(x)$ at the point $x_{0}$,

$$
\vartheta\left(\left|x-x_{0}\right|^{3}\right)=\left.\frac{1}{6} S f\right|_{x_{0}} \cdot\left(x-x_{0}\right)^{3}+\vartheta\left(x-x_{0}\right)^{4} .
$$

Moreover the Schwarzian $\sigma\left(x_{0}\right)=S f\left(x_{0}\right)$ can be used to reconstruct the approximating matrix $M\left(x_{0}\right)$. Replacing $x_{0}$ by the variable $x$, one can use properties (1.3) and (1.4) to verify that

$$
\frac{d M}{d x}=M \cdot\left[\begin{array}{cc}
0 & 1 \\
-\frac{1}{2} \sigma(x) & 0
\end{array}\right]
$$

In other words, the Schwarzian $S f$ measures how far the diffeomorphism $f(x)$ deviates from being the boundary value of some biholomorphism of $\mathbf{U}$. In particular, $f(x)$ is induced by such a biholomorphism if and only if its Schwarzian is identically zero.

A similar type of description can be developed for any bounded domain $\Omega$ whose boundary $\Gamma=b \Omega$ is a smooth curve. To find the correct relation, we shall employ the known existence of a biholomorphism $F: \Omega \rightarrow B$. Working first on the interior of $\Omega$, we define the holomorphic function

$$
h(z)=\left.S F\right|_{z} .
$$

It is actually independent of the choice of the map $F$. To show this, suppose that $F_{1}$ and $F_{2}$ are any two biholomorphisms from $\Omega$ to $B$. The composition $T=F_{2} \circ F_{1}^{-1}$ is a biholomorphism of the unit disk, and as such, must be a linear fractional transformation (with complex coefficients). Because $T$ is the ratio of two linear polynomials, property (1.3) implies that its Schwarzian $S T=0$. Applying the pseudogroup rule (1.4) to the composition $F_{2}=T \circ F_{1}$, it follows that $S F_{2}=S F_{1}$, which is what was claimed about $h(z)$ above. To obtain an invariant along the boundary, we restrict the function $h(z)$ to $\Gamma$ and use the pseudogroup rule to preserve its invariance properties; that is, we define

$$
\gamma_{\Gamma}(s) \equiv S(F \circ \phi)=h(z) \cdot[\dot{\phi}(s)]^{2}+S_{\phi}(s) .
$$

As it stands, $\gamma_{\Gamma}$ is nominally a complex-valued function, however, it is really more special than that. 
FACT 1.12. The special coefficient $\gamma_{\Gamma}$ is a real-valued function.

Using the boundary regularity of $F: \Omega \rightarrow B$ we may define a smooth function $f$ : $\mathbf{R} \rightarrow \mathbf{R}$ via $F(\phi(s))=e^{i f(s)}$. Notice that $f(s)$ is periodic in the sense that $f(s+n L)=$ $f(s)+2 \pi n$ for all integers $n$. Using the pseudogroup property, we may express $\gamma_{\Gamma}$ in terms of the real-valued function $f(s)$,

$$
\gamma=S(F \circ \phi)=S\left(e^{i f}\right)=S\left(e^{i \theta}\right)\left(f^{\prime}\right)^{2}+S f .
$$

But a direct calculation shows that $S\left(e^{i \theta}\right)=\frac{1}{2}$, so that the reality of $\gamma$ is an immediate consequence of the formula

$$
\gamma=\frac{1}{2}\left(f^{\prime}\right)^{2}+S f . \quad \text { Q.E.D. }
$$

The above $f(x)$ is clearly the boundary value of the biholomorphism $F(z)$ as discussed in the main theorem. Next, we investigate its relation to the equation $y^{\prime \prime}+\frac{1}{2} \gamma_{\Gamma} \cdot y=0$.

FACT 1.13. $f(x)=2 \arctan \tau(x) / \beta(x)$ where $\beta$ and $\tau$ satisfy the boundary value problem on the interval $[0, L]$ mentioned in the main theorem.

We begin by examining the role of the arctan function, or rather, its inverse $y=\tan \theta / 2$. Since the latter is a ratio of solutions to the equation $y^{\prime \prime}+\frac{1}{4} y=0$, statement (1.3) implies that the Schwarzian $S(\tan \theta / 2)=\frac{1}{2}$. Using the pseudogroup rule (1.4), the composition $y=\tan \left(\frac{1}{2} f(x)\right)$ satisfies

$$
S(y)=S\left(\tan \frac{1}{2} \theta\right) \cdot\left[f^{\prime}(x)\right]^{2}+S f=\frac{1}{2}\left(f^{\prime}\right)^{2}+S f=\gamma_{\Gamma} .
$$

Another application of (1.3) shows that the function $y: \mathbf{R} \rightarrow \mathbf{R}$ can be written as the ratio of two solutions $\beta(x)$ and $\tau(x)$ to the equation $y^{\prime \prime}+\frac{1}{2} \gamma_{\Gamma} \cdot y=0$. Therefore, we have one of the conditions of the main theorem, namely,

$$
\tan \left[\frac{1}{2} f(x)\right]=\frac{\tau(x)}{\beta(x)} .
$$

Turning to the boundary conditions, recall that the only variable changes which preserve the arclength parameter are the translations $x \rightarrow x+a$. We may select such a transformation so that the boundary function satisfies $f(0)=-\pi$. Because $f(x)$ is a "periodic" diffeomorphism, $f(L)=+\pi$. The previous formula now implies that

$$
\lim _{x \rightarrow 0+} \beta(x)=0 \text { and } \lim _{x \rightarrow L-} \beta(x)=0,
$$

moreover, $\beta(x)$ can never vanish on the interior of $[0, L]$. The derivative of the boundary function is $f(x)=\left[\beta(x)^{2}+\tau(x)^{2}\right]^{-1}$, and because this is a true periodic function, we deduce that $\tau(0)^{2}=\tau(L)^{2} \neq 0$. In order for the ratio $\tau(x) / \beta(x)$ to cover the entire line, the two values $\tau(0)$ and $\tau(L)$ must have opposite signs, hence

$$
\lim _{x \rightarrow 0+} \tau(x)=(-1) \lim _{x \rightarrow L_{-}^{-}} \tau(x) \text {. }
$$

The second derivative $f^{\prime \prime}(x)=-2\left(\beta \beta^{\prime}+\tau \tau^{\prime}\right)\left(\beta^{2}+\tau^{2}\right)^{-2}$ can be used in conjunction with the two previous limit statements to show that

$$
\lim _{x \rightarrow 0+} \tau^{\prime}(x)=(-1) \lim _{x \rightarrow L-} \tau^{\prime}(x)
$$


As the determinant of the Wronskian matrix for $\beta$ and $\tau$ can be chosen to have the constant value one, it now follows that

$$
\lim _{x \rightarrow 0+} \beta^{\prime}(x)=(-1) \lim _{x \rightarrow L_{-}^{-}} \beta^{\prime}(x) \text {. }
$$

Taken together, equations (1.15) to (1.18) are the technical description of the boundary conditions in the main theorem. Q.E.D.

The previous development has been essentially formal in order to expose the role of the special coefficient $\gamma_{\Gamma}$. Next, we employ potential theory in order to identify $\gamma_{\Gamma}$ with a quantity that exists independently of Riemann's mapping function. It is known that Dirichlet's problem for the Laplacian over $\Omega$ has a unique solution; see either [4] or [5]. Consequently, we may assume the existence of the following kernel function.

Definition 1.19. The "bound" Green's function $G(z, w)$ for the domain $\Omega$ satisfies the conditions that

(i) $G(z, w)$ is harmonic in the variable $z \in \Omega-\{w\}$,

(ii) $G(z, w)+\ln |z-w|$ is harmonic for all $z \in \Omega$,

(iii) $G(z, w)=0$ for $w \in \Omega$ and $z \in b \Omega$.

$G(z, w)$ is symmetric in the variables $(z, w) \in \Omega \times \Omega-\Delta$, and it is invariant under holomorphic changes of coordinates. In terms of the bound Green's function, the Bergman kernel is given by

$$
K_{\Omega}(z, \bar{w})=-\frac{2}{\pi} \frac{\partial^{2} G}{\partial z \partial \bar{w}}(z, w) .
$$

It will also be convenient to consider the classical $L$-kernel,

$$
L_{\Omega}(z, w) \equiv-\frac{2}{\pi} \frac{\partial^{2} G}{\partial z \partial w}(z, w) .
$$

This function is holomorphic for $(z, w) \in \Omega \times \Omega-\Delta$, with a double pole along the diagonal, in other words,

$$
L_{\Omega}(z, w)=\frac{1}{\pi} \frac{1}{(z-w)^{2}}-l_{\Omega}(z, w)
$$

where $l_{\Omega}(z, w)$ is holomorphic on the open set $\Omega \times \Omega$ in $\mathbf{C}^{2}$.

Proposition 1.23. If the simply-connected domain $\Omega$ has a smooth boundary $\Gamma$, then $l_{\Omega}(z, w)$ is smooth on $\Gamma \times \Gamma$.

Proof. The argument depends upon the existence of Riemann's mapping function $F: \Omega \rightarrow B$, which may be used to calculate $l_{\Omega}$ in terms of the known data over the unit disk $B$. In fact,

$$
G_{B}(z, w)=\ln \left|\frac{1-z \bar{w}}{z-w}\right|, \quad L_{B}(z, w)=\frac{1}{\pi} \cdot \frac{1}{(z-w)^{2}} \quad \text { and } \quad l_{B}(z, w)=0 .
$$

From the invariance property of the bound Green's function, it follows that the $L$-kernel transforms according to the rule

$$
L_{B}(F(z), F(w)) \cdot F^{\prime}(z) \cdot F^{\prime}(w)=L_{\Omega}(z, w) .
$$


As an immediate consequence, $l_{\Omega}=-(1 / \pi) U_{F}$ where

$$
U_{F}(z, w)=\frac{F^{\prime}(z) F^{\prime}(w)}{[F(z)-F(w)]^{2}}-\frac{1}{(z-w)^{2}} .
$$

By means of elementary calculus, the diagonal values of this function are seen to be

$$
\lim _{w \rightarrow z} U_{F}(z, w)=\frac{1}{6} S F(z) .
$$

This fact makes the potential-theoretic approach useful for the present subject. Because the boundary $\Gamma=b \Omega$ is smooth, a theorem of Carathéodory's implies that $F(z)$ is smooth up to and including, the boundary. Moreover, the derivative $F^{\prime}(z)$ can not vanish on $\Gamma$, for otherwise the image boundary would not be smooth, in contradiction to the fact that $\Gamma$ maps onto $S^{1}$. As the Schwarzian derivative involves $F^{\prime}(z)$ in its denominator, the diagonal values of $l_{\Omega}=-(1 / \pi) U_{F}$ are bounded. Consequently, $l_{\Omega}(z, w)$ is a smooth function of both variables $(z, w) \in \Gamma$ $\times$ T. Q.E.D.

Recall that the boundary is parameterized by a smooth function $\phi: \mathbf{R} \rightarrow \Gamma$ whose basic period $L=$ length $\Gamma$. To simplify subsequent formulae, the boundary values of the Bergman kernel will be denoted by

$$
K_{\Gamma}(s, r) \equiv K_{\Omega}[\phi(s), \overline{\phi(r)}] \phi^{\prime}(s) \overline{\phi^{\prime}(r)} .
$$

This function is known to be smooth on $\Gamma \times \Gamma-\Delta$, with a pole of order two along the diagonal [7].

THEOREM 1.28. The special section $\gamma_{\Gamma}$ can be obtained by the limit statement

$$
\frac{1}{6} \gamma_{\Gamma}(s)=\lim _{r \rightarrow s}\left[-\pi K_{\Gamma}(s, r)-(s-r)^{-2}\right] \text {. }
$$

Proof. Since the bound Green's function vanishes along the boundary and it is symmetric in both variables,

$$
\frac{\partial G}{\partial z}(z, w)=0 \text { for } z \in \Omega, w \in b \Omega
$$

Setting $w=\phi(r)$, and differentiating the composite function,

$$
\frac{\partial^{2} G}{\partial z \partial w}(z, \phi(r)) \phi^{\prime}(r)+\frac{\partial^{2} G}{\partial z \partial \bar{w}}\left(z, \phi^{\prime}(r)\right) \overline{\phi^{\prime}(r)}=0 .
$$

As long as $s \neq r$, this may be evaluated at $z=\phi(s)$ to obtain

$$
L_{\Omega}[\phi(s), \phi(r)] \cdot \phi^{\prime}(s) \cdot \phi^{\prime}(r)+K_{\Gamma}(s, r)=0 .
$$

Now $L_{\Omega}$ is symmetric, and $K_{\Omega}$ is Hermitian symmetric, therefore, both terms in the last equation must be real-valued. By subtracting $(1 / \pi)(s-r)^{-2}$ from both sides, one finds that

$$
U_{\phi}(s, r)+U_{F}(\phi(s), \phi(r)) \cdot \phi^{\prime}(s) \cdot \phi^{\prime}(r)=-\pi K_{\Gamma}(s, r)-1 /(s-r)^{2} .
$$

In view of Proposition 1.23, the diagonal limit of the left-hand side may be 
evaluated directly:

$$
\lim _{r \rightarrow s}\left[U_{\phi}+U_{F} \cdot \phi^{\prime}(s) \cdot \phi^{\prime}(r)\right]=\frac{1}{6}\left\{[S \phi]_{s}+[S F]_{\phi(s)} \cdot\left[\phi^{\prime}(s)\right]^{2}\right\}
$$

However, this is precisely the special coefficient $\gamma_{\Gamma}$ as was defined by means of equations (1.10) and (1.11). Q.E.D.

2. Sufficiency. Since the boundary curve $\Gamma$ is oriented, one may define two real line bundles, the square root of the tangent bundle $T(\Gamma)^{1 / 2}$, whose sections transform according to the rule

$$
X_{\alpha}\left(x_{\alpha}\right)=X_{\beta}\left(h\left(x_{\alpha}\right)\right) \cdot\left[h^{\prime}\left(x_{\alpha}\right)\right]^{-1 / 2},
$$

and the three-halves power of the cotangent bundle $T^{*}(\Gamma)^{3 / 2}$, which is determined by its transformation rule

$$
w_{\alpha}\left(x_{\alpha}\right)=w_{\beta}\left(h\left(x_{\alpha}\right)\right) \cdot\left[h^{\prime}\left(x_{\alpha}\right)\right]^{3 / 2} .
$$

In both cases, the fractional powers are well-defined because the coordinate change $x_{\beta}=h\left(x_{\alpha}\right)$ is orientation preserving. Consider an ordinary differential operator $P$ from the first bundle to the second bundle. Given sections $X$ and $Y$ of $T(\Gamma)^{1 / 2}$, the tensor product $X \otimes P(Y)$ is a differential form, and can be integrated over the curve $\Gamma$. The operator $P$ is said to be formally selfadjoint whenever

$$
\int_{\Gamma} P(X) \otimes Y=\int_{\Gamma} X \otimes P(Y) \text { for all } X \text { and } Y \text { in } C^{\infty}\left(T \Gamma^{1 / 2}\right) \text {. }
$$

If the operator is second-order, its top symbol $\sigma_{P}$ must be a function; hence, the condition $\sigma_{P} \equiv 1$ is an invariant notion. This together with selfadjointness implies that $P$ has no first order term, that is, in every local coordinate

$$
P\left[X \cdot(d / d x)^{1 / 2}\right]=\left[X^{\prime \prime}+\frac{1}{2} c \cdot X\right] \cdot(d x)^{3 / 2} .
$$

The zero-order coefficient is then found to transform according to the rule

$$
c_{\alpha}\left(x_{\alpha}\right)=c_{\beta}\left(h\left(x_{\alpha}\right)\right) \cdot\left[h^{\prime}\left(x_{\alpha}\right)\right]^{2}+S f\left(x_{\alpha}\right) .
$$

By its very definition, the special coefficient $\gamma_{\Gamma}(s)$ transforms according to this rule, thus, it defines a unique operator of the above type. In summary, we have

Proposition 2.6. Associated to the special coefficient $\gamma_{\Gamma}(s)$, there is a unique ordinary differential operator

$$
P_{\Gamma}: \underline{\underline{C}}^{\infty}\left(\Gamma, T^{1 / 2}\right) \rightarrow \underline{\underline{C}}^{\infty}\left(\Gamma, T^{* 3 / 2}\right)
$$

which is second order, formally selfadjoint, and whose top symbol is the constant function one.

This operator is quite special for it possesses an integral inverse whose kernel function can be explicitly identified.

THEOREM 2.7. Green's function $E_{\Gamma}(s, r)$ for $P_{\Gamma}$ is related to the boundary values of Bergman's kernel via

$$
E_{\Gamma}(s, r)=1 / \sqrt{-4 \pi K_{\Gamma}(s, r)}
$$


Proof. The term "Green's function" refers to that distribution over $\Gamma \times \Gamma$ which enjoys the property

$$
\int_{0}^{L} E(s, r) \cdot P f(r) d r=f(s) .
$$

The following conditions will insure that $E(s, r)$ is the correct Green's function; as proof, one need only integrate by parts.

(i) $E$ is symmetric and smooth off the diagonal $\Delta$ in $\Gamma \times \Gamma$;

(ii) as the diagonal is approached,

$$
\lim _{r \rightarrow s} E(s, r)=0, \quad \lim _{r \rightarrow s \pm} \frac{\partial E}{\partial s}(s, r)= \pm \frac{1}{2}
$$

(iii) and along any cross section with constant $r$,

$$
\frac{\partial^{2} E}{\partial s^{2}}(s, r)+\frac{1}{2} \gamma(s) \cdot E(s, r)=0, \quad s \neq r .
$$

It has already been mentioned that $K_{\Gamma}(s, r)$ satisfies the first condition, see the proof of Proposition 1.23. As $K_{\Gamma}$ is strictly negative, the same is true for $\left(-4 \pi K_{\Gamma}\right)^{-1 / 2}$. The second condition follows from the statement of Theorem 1.28 and a simple observation about asymptotic behavior:

FACT 2.10. Suppose that the function $m(x)$ is defined and nonnegative for $x>y$; then, $m(x)=(x-y) / 2+\theta(x-y)^{3}$ if and only if $1 / m(x)^{2}=4 /(x-y)^{2}+$ $\theta(1)$.

To verify the last condition, begin by differentiating the function $k(s)=$ $\left[-4 \pi K_{\Gamma}\right]^{-1 / 2}$ for $s \neq r$, i.e.

$$
\frac{d^{2} k}{d s^{2}}=-\frac{k}{2}\left[-2 \sqrt{K_{\Omega}} \cdot \frac{\partial^{2}}{\partial z^{2}}\left(\frac{1}{\sqrt{K_{\Omega}}}\right) \cdot \phi^{\prime}(s)^{2}+S \phi\right] .
$$

It is well known [2] that the Riemann map $F: \Omega \rightarrow B$ for which $F(w)=0$, has its first derivative given by

$$
F^{\prime}(z)=\alpha \frac{K_{\Omega}(z, \bar{w})}{K_{\Omega}(w, \bar{w})} .
$$

When calculating the Schwarzian, the constant $\alpha$ and the term $K_{\Omega}(w, \bar{w})$ disappear, for by statement (1.2),

$$
S F=-2 \sqrt{K_{\Omega}(z, \bar{w})} \frac{\partial^{2}}{\partial z^{2}}\left(\frac{1}{\sqrt{K_{\Omega}(z, \bar{w})}}\right) .
$$

Shortly, it will be proven that this expression is also independent of the remaining appearance of $w$. In view of formula (1.11), this means that $\left[-4 \pi K_{\Gamma}\right]^{-1 / 2}$ satisfies the final condition (iii). Q.E.D.

FACT 2.14. The right-hand side of expression (2.13) is independent of the parameter $w \in \Omega$.

Proof. The simply-connected domain $\Omega$ equipped with the metric $K_{\Omega}(w, \bar{w})|d w|^{2}$ has constant negative curvature $-4 \pi$; see [2, Chapter III]. There is a standard identity for curvature in terms of the metric tensor 


$$
-4 \pi=\frac{-2}{K(w, w)} \frac{\partial^{2}}{\partial w \partial \bar{w}}[\ln K(w, \bar{w})] .
$$

With the abbreviation $K_{r \bar{s}}=\partial^{2} K_{\Omega} /\left(\partial z^{r} \partial \bar{w}^{s}\right)$, it is useful to consider the following function on $\Omega \times \Omega$,

$$
M(z, \bar{w})=\frac{K(z, \bar{w}) K_{1 \overline{1}}(z, \bar{w})-K_{1 \overline{0}}(z, \bar{w}) K_{0 \overline{1}}(z, \bar{w})}{K(z, \bar{w})^{3}} .
$$

By the curvature remark, $M(z, \bar{w})$ is constant along the diagonal. But repeated use of the chain rule shows that $Q(z)=M(z, \bar{w})-M(w, \bar{w})$ vanishes to infinite order at $z=w$. The identity theorem implies that $Q(z)=0$, which means $M(z, \bar{w})$ is constant on the entire set $\Omega \times \Omega$. Finally, one applies the identity

$$
\frac{\partial}{\partial w}\left[-2 \sqrt{K_{\Omega}} \frac{\partial^{2}}{\partial z^{2}}\left(\frac{1}{\sqrt{K_{\Omega}}}\right)\right]=K_{\Omega} \cdot \frac{\partial M}{\partial z} .
$$

COMMENT 2.15. It is possible to show that

$$
\frac{1}{E_{\Gamma}(s, r)}=-4 \pi i S_{\Omega}(\phi(s), \overline{\phi(r)}) \cdot \sqrt{\phi^{\prime}(s) \overline{\phi^{\prime}(r)}},
$$

where $S_{\Omega}(z, \bar{w})$ is the Szegö kernel for $\Omega$ [8]. The main difficulty is that each of the factors $S_{\Omega}(\phi(s), \overline{\phi(r)})$ and $\sqrt{\phi^{\prime}(s) \overline{\phi^{\prime}(r)}}$ is complex valued and discontinuous along $s=r$. However, they are smooth when $s \neq r$, so that a rehearsal of the previous proof in the presence of the identity $4 \pi S_{\Omega}^{2}=K_{\Omega}$ will establish that the indicated product is real valued, and equal to $E_{\Gamma}^{-1}$. The latter identity can be interpreted as an equality between functions over $\Omega \times \Omega$. It can be proven by noting that it is true for the unit disk, and then invoking the Riemann mapping theorem because both sides transform in the same manner. To avoid Riemann's theorem, one could resort to the potential theory in Chapters V and VII of reference [2].

Comment 2.16. The previous theorem yields a factorization of the orthogonal projection $\pi$ of $L^{2}(\Omega)$ onto the closed subspace $\mathcal{H}^{2}(\Omega)$ of square-integrable holomorphic functions over $\Omega$. This projection is given by integration against the Bergman kernel

$$
\left.\pi \psi\right|_{z}=\iint_{\Omega} K_{\Omega}(z, \bar{w}) \psi(w) \frac{d w \wedge d \bar{w}}{2 i}
$$

The factorization involves two operators. One of them employs a well-known version of the Cauchy kernel,

$$
\left.T h\right|_{z}=\frac{1}{2 \pi i} \int_{b \Omega} \frac{h(w)}{(w-z)^{2}} d w .
$$

This can be viewed as an unbounded operator $T: L^{2}(b \Omega) \rightarrow L^{2}(\Omega)$, so that its adjoint $T^{*}$ maps $L^{2}(\Omega)$ into $L^{2}(b \Omega)$. The other operator is based upon Green's function $E(s, r)$ which was introduced in Theorem 2.7,

$$
\left.\mathcal{L} h\right|_{x}=-\frac{1}{\pi} \int_{s}^{s+L}[\ln E(s, r)] \cdot h(r) d r .
$$


In particular, it is a pseudodifferential operator of order -1 which maps $L^{2}(b \Omega)$ into $L^{2}(b \Omega)$. Using these operators, it is possible to establish the factorization $\pi=T \circ \mathcal{L} \circ T^{*}$ (we omit the proof as this expression will not be employed in the sequel).

We now turn to the central issue of the main theorem. Given a map $f: \Gamma \rightarrow S^{1}$ which satisfies conditions (i) and (ii) of that theorem, the Schwarzian formalism implies that

$$
\gamma=\frac{1}{2} \cdot\left(f^{\prime}\right)^{2}+S f .
$$

By the pseudogroup rule, the functional inverse $g: S^{1} \rightarrow \Gamma$ must satisfy the relation

$$
\frac{1}{2}=\gamma \cdot\left(g^{\prime}\right)^{2}+S g \text {. }
$$

At this point, we utilize the special geometric nature of the operators $P_{S^{1}}$ and $P_{\Gamma}$. The transformation rule (2.18) means that under the coordinate change $s=g(\theta)$, the operator $P_{\Gamma}$ is converted into the operator $P_{S^{1}}$. Correspondingly, the integral inverse for $P_{\Gamma}$ goes into the integral inverse for $P_{S^{1}}$. On the basis of conditions (i) through (iii) of Theorem 2.7, we find that

$$
E_{\Gamma}(g(\theta), g(\phi)) \cdot\left[g^{\prime}(\theta) g^{\prime}(\phi)\right]^{-1 / 2}=E_{S^{1}}(\theta, \phi) .
$$

The major identity of Theorem 2.7 now implies that the boundary values of the respective Bergman kernels will be related via

$$
K_{\Gamma}(g(\theta), g(\phi)) \cdot g^{\prime}(\theta) \cdot g^{\prime}(\phi)=K_{S^{1}}(\theta, \phi) .
$$

This will be employed in the proof of the next statement.

TheOREM 2.20. The "periodic" diffeomorphism $g:[0,2 \pi] \rightarrow[0, L]$ can be extended to a biholomorphism $G: B \rightarrow \Omega$ such that

$$
G\left(e^{i \theta}\right)=\phi[g(\theta)]
$$

Proof. Let $\mathscr{Q}^{2}\left(S^{1}\right)$ denote the closed subspace of $L^{2}\left(S^{1}\right)$ generated by the functions $\left\{e^{i n \theta}: n=0,1,2, \ldots\right\}$; it is well known that the elements of this subspace can be extended to holomorphic functions on $B$. Further let $Q: L^{2}\left(S^{1}\right) \rightarrow$ $\mathcal{Q}^{2}\left(S^{1}\right)$ be the orthogonal projection, and let $Q^{\perp}$ be the projection onto the orthogonal complement $\mathcal{Q}^{2}\left(S^{1}\right)^{\perp}$ which is generated by the antiholomorphic functions $\left\{e^{-i n \theta}: n=1,2, \ldots\right\}$. In this language, we must show that $\tilde{g}\left(e^{i \theta}\right) \equiv$ $\phi[g(\theta)]$ satisfies $Q^{\perp} \tilde{g}=0$. Notice that the operator $D=-i(\partial / \partial \theta)$ is symmetric and unbounded on $L^{2}\left(S^{1}\right)$; moreover, it is strict negative definite on $\mathcal{U}^{2}\left(S^{1}\right)$-just check its action on the basis $\left\{e^{-i n \theta}\right\}$. Consequently,

$$
0 \geqslant\left\langle D Q^{\perp} \tilde{\boldsymbol{g}}, Q^{\perp} \tilde{\mathrm{g}}\right\rangle,
$$

and it suffices to prove that this number is actually zero. With this objective in mind, we rewrite the above expression.

LEMMA 2.21 .

$$
\left\langle D Q^{\perp} \tilde{g}, Q^{\perp} \tilde{g}\right\rangle=\operatorname{area} \Omega+\frac{1}{4} \iint_{S^{1} \times S^{1}}|\tilde{g}(z)-\tilde{g}(w)|^{2} K_{B}(z, \bar{w}) d z \wedge d \bar{w}
$$


This depends upon our explicit knowledge of the projection $Q$. Given any $f \in L^{2}\left(S^{1}\right)$, Cauchy's integral formula yields a holomorphic function on the interior,

$$
F(z)=\frac{1}{2 \pi i} \int_{S^{\prime}} \frac{f(w) d w}{w-z}=\frac{1}{2 \pi} \int_{0}^{2 \pi} \frac{f\left(e^{i \phi}\right) d \phi}{1-z e^{-i \phi}} .
$$

But when do the boundary values of $F$ agree with the values of the original function $f\left(e^{i \phi}\right)$ ? On the one hand, the evaluation of $F(z)$ at $z=e^{i \theta}$ yields a singular integral equation

$$
F\left(e^{i \theta}\right)=\frac{1}{2}\left[\int_{0}^{2 \pi}\left(f e^{i \theta}\right) \frac{d \theta}{2 \pi}+\frac{i}{2 \pi} \lim _{\varepsilon \rightarrow 0} \int_{\varepsilon<|\phi|<\pi} f(\phi) \operatorname{ctn}\left(\frac{\theta-\phi}{2}\right) d \phi\right] .
$$

We shall denote the operator in square brackets by $Z=M+i C$. An examination of its behavior on the trigonometric basis $\left\{e^{i k \theta}: k \in \mathbf{Z}\right\}$ reveals that $Z$ is selfadjoint and satisfies $Z^{2}=I$ (see reference [12] where the operator $C$ is studied in detail). On the other hand, there is a classical formula of Plemelj, see reference [10], which uses the boundary behavior of single- and double-layer potentials to deduce that

$$
\lim _{r \rightarrow 1-} F\left(r e^{i \theta}\right)=\left[\frac{1}{2}(I+Z) f\right]_{\theta} .
$$

This means that $Q=\frac{1}{2}(I+Z)$ because both operators are selfadjoint, and both are the identity on the subspace $\mathcal{Q}^{2}\left(S^{1}\right)$. Similarly one has $Q^{\perp}=\frac{1}{2}(I-Z)$, and the quadratic form which interests us is given by

$$
\left\langle D Q^{\perp} \tilde{g}, Q^{\perp} \tilde{g}\right\rangle=\frac{1}{4}\{\langle D \tilde{g}, \tilde{g}\rangle-\langle D Z \tilde{g}, \tilde{g}\rangle-\langle D \tilde{g}, Z \tilde{g}\rangle+\langle D Z \tilde{g}, Z \tilde{g}\rangle\} .
$$

Now $Z$ commutes with $D$ because it is a convolution operator. Further, since $Z$ is selfadjoint and $Z^{2}=I$, the previous expression reduces to

$$
\left\langle D Q^{\perp} \tilde{g}, Q^{\perp} g\right\rangle=\frac{1}{2}\langle D \tilde{g}, \tilde{g}\rangle-\frac{1}{2}\langle D Z \tilde{g}, \tilde{g}\rangle .
$$

Since $\tilde{g}$ encircles the interior of $\Omega$ in the positive sense as $\theta$ varies from 0 to $2 \pi$, Stokes's theorem can be applied to evaluate the first term,

$$
\frac{1}{2}\langle D \tilde{g}, \tilde{g}\rangle=\frac{1}{2} \int_{b \Omega}(x d y-y d x)=\text { area } \Omega .
$$

Recall that $Z=M+i C$ where $M$ transforms any function into its mean value. Because $M \tilde{g}$ is a constant function, $D M \tilde{g}=0$, and so the second term reduces to

$$
-\frac{1}{2}\langle D Z \tilde{g}, \tilde{g}\rangle=-\frac{i}{2}\langle D C \tilde{g}, \tilde{g}\rangle=-\frac{1}{2}\left\langle\frac{d}{d s} C \tilde{g}, \tilde{g}\right\rangle \text {. }
$$

Decomposing $g=g_{1}+i g_{2}$, and noting that $C$ is a real, skew-symmetric operator that commutes with differentiation,

$$
\left\langle\frac{d}{d s} C \tilde{g}, \tilde{g}\right\rangle=\left\langle\frac{d}{d s} C g_{1}, g_{1}\right\rangle+\left\langle\frac{d}{d s} C g_{2}, g_{2}\right\rangle
$$

Both of the latter terms can be handled in the same manner; we shall only employ the fact that $g_{1}$ and $g_{2}$ are smooth (because $\tilde{g}$ was constructed from solutions of an ordinary differential equation). In other words, it suffices to consider the symmetric form $\langle(d / d s) C h, h\rangle$ where $h$ is a real-valued, periodic, and 
differentiable function on $[0,2 \pi]$. The periodicity means that we can integrate by parts without introducing boundary terms

$$
\left\langle\frac{d}{d s} C h, h\right\rangle=(-1)\left\langle C h, \frac{d h}{d s}\right\rangle \text {. }
$$

The operator $C$ is given by convolution against the function $\operatorname{ctn}(\theta / 2)$. As this has mean value zero, the principal value expression for $C h$ may be replaced by a Riemann integral

$$
\left.C h\right|_{x}=\frac{1}{2 \pi} \int_{0}^{2 \pi}[h(y)-h(x)] \operatorname{ctn}\left(\frac{x-y}{2}\right) d y .
$$

This allows one to interchange the order of integration in $(-1)\langle C h, d h / d s\rangle$, and study the expression

$$
J(y) \equiv \int_{y}^{2 \pi+y}\left[-h^{\prime}(x)\right][h(y)-h(x)] \operatorname{ctn}((x-y) / 2) d x
$$

Using $H(x)=h(y)-h(x)$ as an indefinite integral for $-h^{\prime}(x)$, a further integration by parts gives

$$
2 J(y)=\left.H(x) \operatorname{ctn}\left(\frac{x-y}{2}\right)\right|_{y} ^{2 \pi+y}+\frac{1}{2} \int_{y}^{2 \pi+y} H(x)^{2} \csc ^{2}\left(\frac{x-y}{2}\right) d x .
$$

However the boundary term vanishes, so this calculus argument yields

$$
\left\langle\frac{d}{d s} C h, h\right\rangle=\frac{1}{8 \pi} \int_{0}^{2 \pi} \int_{0}^{2 \pi} \frac{[h(x)-h(y)]^{2}}{\sin ^{2}((x-y) / 2)} d x d y .
$$

Returning to the second term of expression (2.24) for our original quadratic form, we have obtained

$$
-\frac{1}{2}\langle D Z \tilde{g}, \tilde{g}\rangle=-\frac{1}{16 \pi} \int_{0}^{2 \pi} \int_{0}^{2 \pi} \frac{\left|\tilde{g}\left(e^{i \theta}\right)-\tilde{g}\left(e^{i \theta}\right)\right|^{2}}{\sin ^{2}((\theta-\phi) / 2)} d \theta d \phi .
$$

(This is what Douglas employed in [6].) To give it a more invariant expression, we notice that the Bergman kernel for the unit disk is $K_{B}(z, \bar{\omega})=(1 / \pi)(1-z \bar{\omega})^{-2}$, and restricting it to the boundary $K_{S^{\prime}}(x, y)=-(1 / 4 \pi) \csc ^{2}((x-y) / 2)$. Finally by converting to the notation of differential forms,

$$
-\frac{1}{2}\langle D Z \tilde{g}, \tilde{g}\rangle=\frac{1}{4} \iint_{S^{1} \times S^{1}}|\tilde{g}(z)-\tilde{g}(\omega)|^{2} K_{B}(z, \bar{\omega}) d z \wedge d \bar{\omega} .
$$

This completes the proof of Lemma 2.21, so we may now resume the proof of Theorem 2.20.

The final argument hinges upon our ability to change variables in the second term of the new expression for $\left\langle D Q^{\perp} \tilde{\boldsymbol{g}}, Q^{\perp} \tilde{\boldsymbol{g}}\right\rangle$. Notice that in equation (2.27) we have $\tilde{g}(z)=\phi \circ g(\theta)$ when $z=e^{i \theta}$, and $\tilde{g}(\omega)=\phi \circ g(\phi)$ where $\omega=e^{i \phi}$. By construction, $g$ was the functional inverse of $f: \Gamma \rightarrow S^{1}$, so we may convert (2.27) into an integral over $\Gamma \times \Gamma$ by means of the substitution $\theta=f(s)$ and $\phi=f(r)$. However, by the remark preceding Theorem 2.20 , the boundary values $K_{B}(z, \bar{\omega}) d g$ $\wedge d \bar{\omega}$ are transformed precisely into the boundary values $K_{\Omega}(\zeta, \bar{\xi}) d \zeta \wedge d \bar{\xi}$. Consequently, the quadratic form becomes 


$$
\left\langle D Q^{\perp} \tilde{g}, Q^{\perp} \tilde{g}\right\rangle=\operatorname{area} \Omega+\frac{1}{4} \iint_{\Gamma \times \Gamma}|\zeta-\xi|^{2} K_{\Omega}(\zeta, \bar{\xi}) d \zeta \wedge d \bar{\xi},
$$

and we can now show directly that the above expression is zero. The Bergman kernel $K_{\Omega}(\zeta, \bar{\xi})$ may be written as an infinite series $\Sigma_{1}^{\infty} \phi_{n}(\zeta) \overline{\phi_{n}(\xi)}$ where $\left\{\phi_{n}(\zeta)\right\}$ is any orthonormal basis for $\mathcal{H}^{2}(\Omega)$, the space of square-integrable holomorphic functions on $\Omega$. Select such a basis whose first member is the constant function $\phi_{1}(\zeta)=(\text { area } \Omega)^{-1 / 2}$. Because the factor $|\zeta-\xi|^{2}$ compensates for the pole of the Bergman kernel along the diagonal of $\Gamma \times \Gamma$, the integral in (2.28) can be expressed as an infinite sum,

$$
\sum_{n=1}^{\infty} \iint_{\Gamma \times \Gamma}|\zeta-\xi|^{2} \phi_{n}(\zeta) \overline{\phi_{n}(\zeta)} d \zeta \wedge d \bar{\xi}
$$

We examine each term separately. By expanding the factor $|\zeta-\xi|^{2}$, we obtain four new integrals, which can be rewritten by means of Fubini's theorem,

$$
\begin{gathered}
\iint_{\Gamma \times \Gamma} \zeta \bar{\zeta} \phi_{n}(\zeta) \overline{\phi_{n}(\xi)} d \zeta \wedge d \bar{\xi}=\int_{\Gamma}|\zeta|^{2} \cdot \phi_{n}(\zeta) d \zeta \cdot \overline{\int_{\Gamma} \phi_{n}(\xi) d \xi} \\
-1 \cdot \iint_{\Gamma \times \Gamma} \zeta \bar{\xi} \cdot \phi_{n}(\zeta) \overline{\phi_{n}(\xi)} d \zeta \wedge d \bar{\xi}=(-1)\left|\int_{\Gamma} \zeta \phi_{n}(\zeta) d \zeta\right|^{2} \\
(-1)^{2} \iint_{\Gamma \times \Gamma} \bar{\xi} \phi_{n}(\zeta) \overline{\phi_{n}(\xi)} d \zeta \wedge d \bar{\xi}=\int_{\Gamma} \phi_{n}(\zeta) d \zeta \cdot \int_{\Gamma}|\xi|^{2} \phi_{n}(\xi) d \xi \\
-1 \cdot \iint_{\Gamma \times \Gamma} \overline{\zeta \xi} \cdot \phi_{n}(\zeta) \overline{\phi_{n}(\xi)} d \zeta \wedge d \bar{\xi}=(-1)\left|\int_{\Gamma} \overline{\zeta \phi_{n}}(\zeta) d \zeta\right|^{2}
\end{gathered}
$$

The functions $\phi_{n}(z)$ and $z \phi_{n}(z)$ are holomorphic inside $\Gamma$, hence, Cauchy's theorem implies that the first three terms vanish. The final term can be handled by Stoke's theorem,

$$
\int_{\Gamma} \bar{\zeta} \phi_{n}(\zeta) d \zeta=\iint_{\Omega} \phi_{n}(\zeta) d \bar{\zeta} d \zeta
$$

When $n \geqslant 2$, this is zero because $\phi_{n}(\zeta)$ is orthogonal to the constant function $\phi_{1}(\zeta)$. When $n=1$, it becomes $2 i \sqrt{\text { area } \Omega}$. All together, these calculations give

$$
\iint_{\Gamma \times \Gamma}|\zeta-\xi|^{2} K_{\Omega}(\zeta, \bar{\xi}) d \zeta \wedge d \bar{\xi}=(-1)\left|\int_{\Gamma} \bar{\zeta}_{1}(\zeta) d \zeta\right|^{2}=-4 \text { area } \Omega .
$$

Thus we have proven that the quadratic form $\left\langle D Q^{\perp} \tilde{g}, Q^{\perp} \tilde{g}\right\rangle=0$. By our earlier remarks, this means that $\tilde{g}(z)=\phi[g(\theta)]$ possesses a holomorphic extension $G$ to the interior of the unit disk $B$. It is allowable to apply the residue theorem to $G(z)$ over the unit circle; because its boundary value $\tilde{g}$ encircles the domain $\Omega$ in the positive sense, we conclude that $G(z)$ maps $B$ bijectively onto $\Omega$. Q.E.D.

Appendix. In view of the role played by the special section $\gamma_{\Gamma}$, it is reasonable to ask about its relation to the curvature function $K_{\Gamma}(s)$ of the boundary. One might conjecture that $\gamma_{\Gamma}$ depends in a differential manner upon $K_{\Gamma}$, more precisely, that there is some integer $n$ and some function $f\left(s, x_{0}, \ldots, x_{n}\right)$ such that 


$$
\gamma_{\Gamma}(s)=f\left(s, K(s), \frac{d K}{d s}(s), \ldots, \frac{d^{n} K}{d s^{n}}(s)\right) .
$$

Were this true, a small change in the curvature function near one point would not effect the value of $\gamma_{\Gamma}$ near other points on the curve. To pursue this idea, select a curve $\Gamma_{e}$ which is a small perturbation of the unit circle; for $\rho(\theta) \in C_{0}^{\infty}(\mathbf{R})$ with support in some small interval $(-\delta, \delta)$, define this curve by

$$
\psi_{e}(\theta)=[1+\varepsilon \rho(\theta)] e^{i \theta}, \quad-\pi<\theta<\pi .
$$

Notice that $\Gamma_{\varepsilon}$ will coincide with $S^{1}$ for $\delta<|\theta|<\pi$.

ClaIm. The special section of the above curve satisfies

$$
\gamma_{\Gamma}(\theta)=\frac{1}{2}-\varepsilon \frac{6}{\pi} \int_{-\pi}^{\pi} \frac{\rho(t) d t}{|\psi(\theta)-\psi(t)|^{4}}+\sigma(\varepsilon)
$$

whenever $\delta<|\theta| \leqslant \pi$.

REMARK. Recall that the special section for the unit circle is $\gamma_{S^{1}}=\frac{1}{2}$. By choosing $\varepsilon$ so small that the integral term dominates the error term, it is seen that $\gamma_{\Gamma_{\varepsilon}} \neq \gamma_{S^{1}}$ for all points on the common arc $\Gamma_{e} \cap S^{1}$. This is contrary to what one would expect were $\gamma_{\Gamma}$ to depend differentially upon $K_{\Gamma}$, hence the conjectured equation (A.1) cannot be true.

Proof. Let $\Omega_{\varepsilon}$ be the domain interior to $\Gamma_{e}$, and let $G_{\varepsilon}$ be the associated bound Green's function. In particular, $G_{0}=\ln (|1-z w| /|z-w|)$ is the function for the unit disk. Several texts, for example, [2] or [9], discuss Hadamard's variational formula. It can be applied to describe the change of $G_{e}$ in terms of $G_{0}$. Because Bergman's kernel $K_{\varepsilon}$ is a second derivative of $G_{\varepsilon}$, Hadamard's procedure implies that

$$
K_{\varepsilon}(z, \bar{w})=K_{0}(z, \bar{w})+\varepsilon \int_{-\pi}^{\pi} K_{0}\left(z, e^{-i t}\right) \cdot K_{0}\left(e^{i t}, \bar{w}\right) \rho(t) d t+o(\varepsilon)
$$

This may be evaluated at distinct points $z=\psi(\theta)$ and $w=\psi(\theta)$ on the common $\operatorname{arc} \Gamma_{e} \cap S_{1}$. By Theorem 1.28, the passage to the limit $\phi \rightarrow \theta$ produces

$$
\gamma_{\Gamma}(\theta)=\gamma_{S_{1}}(\theta)-6 \pi \varepsilon \int_{-\pi}^{\pi}\left|K_{0}(\psi(\theta), \overline{\psi(t)})\right|^{2} \rho(t) d t+o(\varepsilon)
$$

From the explicit description of Bergman's kernel on the unit disk, the proof is completed by verifying that $\left|K_{0}(z, \bar{w})\right|^{2}=\pi^{-2}|z-w|^{-4}$ for $z \neq w$ on $S^{1}$. Q.E.D.

\section{BIBLIOGRAPHY}

1. L. V. Ahlfors, Conformal invariants, McGraw-Hill, New York, 1973.

2. S. Bergman, The kernel function and conformal mapping, Math. Surveys, no. 5, Amer. Math. Soc., Providence, R. I., 1950.

3. S. S. Chern and J. K. Moser, Real hypersurfaces in complex manifolds, Acta Math. 133 (1974), 219-271.

4. R. Courant, Dirichlet's principle, Interscience, New York, 1950.

5. R. Courant and D. Hilbert, Methods of mathematical physics. II, Interscience, New York, 1962.

6. J. Douglas, Solution of the problem of Plateau, Trans. Amer. Math. Soc. 33 (1931), 263-321. 
7. N. Kerzman, The Bergman kernel function. Differentiability at the boundary, Math. Ann. 195 (1972), 149-158.

8. N. Kerzman and E. M. Stein, The Cauchy kernel, the Szegö kernel, and the Riemann mapping function, Math. Ann. 236 (1978), 85-93.

9. Z. Nehari, Conformal mapping, McGraw-Hill, New York, 1952.

10. N. I. Muskhelishvili, Singular integral equations, Noordhoff, Groningen, 1953.

11. S. Sternberg, Lectures on differential geometry, Prentice-Hall, Englewood Cliffs, N. J., 1964.

12. A. Zygmund, Trigonometric series, 2nd ed., Cambridge Univ. Press, London and New York, 1959.

Department of Mathematics, Duke University, Durham, North Carolina 27706 